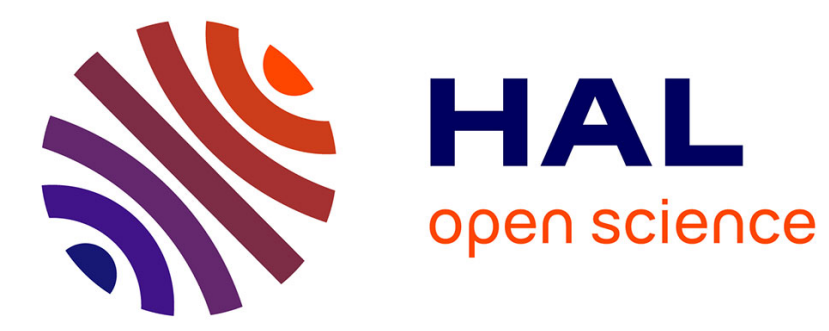

\title{
Les collections, nouvel atout des bibliothèques
}

Fabienne Henryot

\section{To cite this version:}

Fabienne Henryot. Les collections, nouvel atout des bibliothèques. Culture et Musées, 2021, 37, pp.135-162. 10.4000/culturemusees.6343 . hal-03274376

\section{HAL Id: hal-03274376 https://hal.science/hal-03274376}

Submitted on 30 Jun 2021

HAL is a multi-disciplinary open access archive for the deposit and dissemination of scientific research documents, whether they are published or not. The documents may come from teaching and research institutions in France or abroad, or from public or private research centers.
L'archive ouverte pluridisciplinaire HAL, est destinée au dépôt et à la diffusion de documents scientifiques de niveau recherche, publiés ou non, émanant des établissements d'enseignement et de recherche français ou étrangers, des laboratoires publics ou privés. 


\title{
OpenEdition
}

Journals

\section{Culture \& Musées}

Muséologie et recherches sur la culture

$37 \mid 2021$

Les collections patrimoniales ont-elles un avenir ?

Dossier

\section{Les collections, nouvel atout des bibliothèques}

\author{
Libraries' Collections, a Burgeoning Asset
}

Las colecciones: ¿el nuevo acervo de las bibliotecas

\section{Fabienne Henryot}

p. $135-162$

https://doi.org/10.4000/culturemusees.6343

\section{Résumés}

Français English Español

Les bibliothèques publiques dépositaires de collections patrimoniales sont aujourd'hui incitées à inscrire leurs actions en faveur de ces collections dans le cadre général de politiques définies par l'État. En même temps, elles doivent répondre de leur utilité auprès des collectivités qui les financent. L'étude conjointe des politiques incitatives de l'État depuis douze ans, et des textes programmatiques qui fixent l'engagement des bibliothèques dans le cadre plus large d'une politique municipale ou intercommunale, montre que les bibliothèques utilisent les collections pour obtenir une reconnaissance d'une légitimité culturelle et scientifique qui leur fait encore défaut. Ce faisant, elles apprennent à se penser comme des objets politiques.

Public libraries housing national collections are today urged to focus their projects concerning these collections on state defined policies. At the same time, from a utilitarian standpoint, they are accountable to those administrations that provide them with financial contributions. A joint survey of state incentive policies of the past twelve years and documents defining libraries' commitments within the wider field of local policies reveals that libraries take advantage of their collections in order to obtain recognition the scientific and cultural legitimacy to which they aspire. They thus begin to consider themselves political objects.

Las bibliotecas públicas que poseen colecciones patrimoniales, son invitadas en el presente a inscribir sus acciones en favor de esas colecciones en el marco general de las políticas definidas por el Estado. Al mismo tiempo, deben rendir cuentas sobre su utilidad a las instituciones que las financian. Un estudio conjunto de las políticas de incentivo del Estado desde hace doce años, y de los textos programáticos que establecen el compromiso de las bibliotecas en el marco más amplio de 
una política municipal o intermunicipal, muestra que las bibliotecas utilizan las colecciones para obtener el reconocimiento de una legitimidad cultural y científica de la que aún carecen. Al hacerlo, aprenden a pensar en sí mismas como objetos políticos.

\section{Entrées d'index}

Mots-clés : bibliothèques publiques, patrimoine, politiques culturelles, conservation

Keywords: public libraries, heritage, cultural policies, curation

Palabras clave: bibliotecas públicas, patrimonio, políticas culturales, conservación

\section{Notes de la rédaction}

Manuscrit reçu le 15 avril 2020

Version révisée reçue le 2 juillet 2020

Article accepté pour publication le 28 septembre 2020

\section{Texte intégral}

Le titre de cet article est évidemment provocateur, tant la bibliothèque se définit traditionnellement par ses collections et par leur organisation intellectuelle et topographique. Il n'y a pas de bibliothèque sans collection minimale. Pourtant, une rapide revue de la littérature professionnelle, en particulier de l'emblématique Bulletin des bibliothèques de France ${ }^{1}$, montre que les collections sont passées relativement au second plan dans le champ sémantique où s'inscrit la bibliothèque. Depuis dix ans, seulement $23 \%$ des articles sont consacrés aux collections et aux politiques documentaires. À l'inverse, les services en tous genres, accessibilité, confort, accueil, formation, et l'inventivité en matière de médiation culturelle sont le sujet de respectivement $31 \%$ et $34 \%$ des articles. La bibliothèque est donc devenue un espace physique ou virtuel de services et d'animations aux fins de susciter du lien social, de réduire les inégalités culturelles et, plus généralement, sociales (Lachal, 2019) : cette fameuse " bibliothèque troisième lieu » qui semble aujourd'hui faire l'unanimité (Servet, 2010). Face à des actions culturelles de plus en plus déconnectées des fonds documentaires, face à l'installation de cellules de Pôle emploi ou de guichets d'écrivains publics dans les bibliothèques, à quoi servent encore les collections ? Si la fréquentation des espaces reste au beau fixe, les prêts sont en baisse (Evans et al., 2013), preuve que dans les représentations et les pratiques des usagers - qui ose dire aujourd'hui « des lecteurs » ? -, la bibliothèque n'est plus que très lointainement le lieu de déploiement d'une collection.

2 La rupture n'est évidemment pas aussi nette dans les bibliothèques patrimoniales, où les collections constituent le fondement de l'attractivité des établissements, un document n'étant presque jamais équivalent à un autre qui se trouverait ailleurs. Ceci dit, nombre de bibliothèques patrimoniales tentent désormais de créer aussi des liens avec des publics non érudits en comptant sur le pouvoir de séduction de l'« ancien », du " rare » et du «précieux »- les trois qualificatifs conventionnels des fonds patrimoniaux dans les

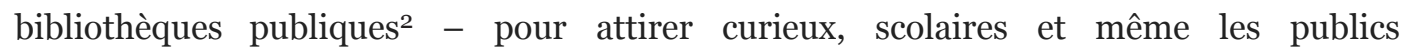
ordinairement éloignés de la culture. Le patrimoine est désormais observé et instrumentalisé comme un vecteur de démocratisation culturelle (Foucher, 2018), position qui tient à distance les collections, remplacées par des « contenus », des images, des artefacts, des dispositifs ludiques.

3 Ce clivage continu entre collections et documents d'une part, et services et médiation d'autre part, n'est évidemment pas insurmontable. Mais il est aggravé par l'ambiguïté profonde qui entoure la fonction, voire l'identité institutionnelle de la bibliothèque depuis sa refondation il y a deux siècles (Varry, 1991 ; Poulain, 1992 ; Kupiec, 1997) : elle est, selon le vœu des administrations révolutionnaire puis impériale, un lieu d'éducation du citoyen et de normalisation d'une pensée jugée éclairée, et non pas une institution patrimoniale comme le sont originellement les musées et les services d'archives. Cette 
injonction éducative explique que les fonds patrimoniaux, entièrement hérités d'un Ancien Régime servant de repoussoir à la modernité, aient été littéralement ensevelis sous les collections courantes et laissés localement à l'abandon depuis leur agrégation dans les bibliothèques publiques en 1803, à l'exception des grandes bibliothèques parisiennes ou provinciales (Varry, 1990 ; Oddos, 1997). La remise en ordre est encore récente, en tout cas, non antérieure au début des années 1990 ; elle est, aussi, très progressive et imparfaitement aboutie à ce jour.

$4 \quad$ C'est sur cette réappropriation que cet article s'interroge, en analysant les moteurs, les acteurs et la chronologie de cette archéologie des collections surgie - et ce n'est pas un hasard - peu après les commémorations des événements de 1789, la Révolution étant identifiée (à tort) comme un temps de redistribution et d'équité culturelle dans le domaine de la lecture et du savoir, lorsque les livres saisis dans les maisons religieuses et chez les aristocrates en émigration ont été destinés aux premières collections publiques (Varry, 1989). Sans forcer le trait du caractère opportuniste de cette réappropriation que suggère notre titre, et sans minimiser non plus les contraintes qu'elles représentent pour les bibliothèques qui en conservent, il s'agit bien de comprendre les avantages que procurent les collections patrimoniales - en termes d'image, de visibilité, de positionnement politique, social et culturel - et, ce faisant, les attributions politiques, sociales et culturelles du patrimoine des bibliothèques dans ces équipements de la culture. Pour déterminer ces avantages et ces attributions, nous interrogeons successivement les deux pôles auprès desquels les bibliothèques ont à la fois des comptes à rendre et une reconnaissance politique et culturelle à négocier. Ce sont l'État, producteur de politiques incitatives en faveur du patrimoine des bibliothèques et instance de contrôle des bibliothèques de tout statut d'une part, et les collectivités territoriales dont dépendent les bibliothèques municipales ou intercommunales d'autre part. Cette comparaison doit permettre de saisir la place attribuée aux collections par les bibliothèques dès lors que ces institutions sont en recherche de légitimation.

5 De cette manière, cet article entend contribuer à l'histoire récente, encore méconnue, du patrimoine écrit - pour employer une expression commode (Henryot, 2019) - comme catégorie d'intervention publique à différentes échelles, et le levier que constituent, dans ces politiques culturelles, les collections patrimoniales des bibliothèques.

\section{Un temps de redécouverte? Les politiques nationales à l'égard du patrimoine des bibliothèques}

$6 \quad$ Sans entrer dans une histoire détaillée des injonctions et des moyens mis par l'État dans la curation des collections patrimoniales des bibliothèques, l'année 2004 constitue à n'en pas douter une rupture. L'État et les bibliothèques municipales ont renoué des liens jusqu'alors distendus grâce au Plan d'action pour le patrimoine écrit (désormais PAPE), lancé par le ministère de la Culture en 2004. Ce plan, s'il entend initier une reprise en main concertée des fonds patrimoniaux, est aussi le résultat d'une réflexion née au milieu des années 1970, qui commençait à prendre la mesure des carences de conservation et de signalement qui affectaient ces fonds. Le positionnement de l'État à l'égard des bibliothèques municipales est en effet délicat, puisque celles-ci conservent des fonds au statut juridique hétérogène : fonds acquis sur budget municipal, fonds d'État entrés par séquestre $(1791,1901)$, voire dépôts de collections privées. Les bibliothèques municipales qui conservent de tels fonds ont ainsi des comptes à rendre à l'État, mais n'ont pas toujours matériellement les moyens de le faire. Du reste, depuis la loi du 22 juillet 1983 , toutes les bibliothèques territoriales sont placées sous le regard de l'État, qu'elles conservent ou non des fonds nationaux (Gautier-Gentès, 1998). 
7 Différents rapports avaient tiré la sonnette d'alarme : en 1975, le rapport de Françoise Bléchet et d'Annie Charon avait dénombré dans les bibliothèques municipales, universitaires et des grands établissements, deux millions d'imprimés d'avant 1800 encore à cataloguer - chiffre très sous-estimé. En 1982, le rapport de Louis Desgraves avait souligné l'état lamentable de nombreuses collections, affirmant qu'en l'absence de mesures, « ce n'est plus un plan de sauvegarde qu'il faudra mettre en place, mais un plan de reconstitution des collections nationales, à supposer que les pertes prévisibles ne soient pas irréparables » (Desgraves, 1982). En 1984, le rapport Yvert préconisait la création de services régionaux de coopération pour les bibliothèques. Mais les lois de décentralisation de 1982 et 1983 n'avaient pas permis de faire émerger le patrimoine des bibliothèques dans les priorités des collectivités locales, faute d'une distribution efficace des compétences culturelles. Cette incurie est souvent expliquée par le caractère quasiment dogmatique de la lecture publique dans les politiques en faveur du livre depuis la fin du xixe siècle, et plus encore depuis les années 1930 (Lassalle, 1997). Le rôle moteur joué par la Bibliothèque nationale de France dans la coopération documentaire à partir de 1996, l'instauration du dépôt légal imprimeur auprès de 24 bibliothèques territoriales la même année, les débuts de la numérisation de masse en bibliothèque à partir de 1996 également, enfin la mise en place du Catalogue collectif de France en 1997 ont rappelé aux municipalités l'existence de fonds anciens riches mais méconnus des agents mêmes qui en avaient la charge, et l'urgence de les sécuriser et de les inventorier (Tesnière, 2006).

8 Dans ce contexte brossé à grands traits, le PAPE devait impulser une dynamique au sein des régions, et coordonner les actions en s'assurant de leur convergence d'un point de vue national. Le PAPE s'appuyait ainsi sur la dynamique régionale formalisée en 2003. L'État se réservait uniquement - et se réserve toujours - la conception des grandes orientations du plan, laissant le soin aux Régions de les adapter localement en fonction des priorités. Celles-ci sont au nombre de cinq : la conservation et l'enrichissement des collections nationales ; la constitution des ressources d'information ; la mise en valeur des collections ; l'élargissement des lecteurs et des publics ; la formation des personnels (Cohen \& Yvon, 2004). Au terme d'une phase de diagnostic (2004-2007) est remontée à la surface une réalité patrimoniale d'environ 50 millions de documents, dont la moitié à la $\mathrm{BnF}$, l'autre répartie entre les 350 à 400 bibliothèques territoriales. Cette masse documentaire souffrait de nombreuses carences : identification imprécise de la propriété des collections ; conservation le plus souvent incohérente et menée sans priorisation ( $31 \%$ des collections en mauvais état) ; sécurisation inexistante des collections dans la moitié des établissements ; catalogage, voire inventaire insuffisants (36\% des collections n'étaient pas du tout signalées, même sommairement). Plus grave, la notion même de patrimoine ne faisait pas consensus parmi les professionnels des bibliothèques, pas plus qu'il n'avait, en conséquence, de place clairement assignée dans la politique de la bibliothèque et dans celle de sa tutelle (Plazannet, 2008 ; Loiret, 2014).

$9 \quad$ Le ministère de la Culture propose annuellement depuis 2007 un appel à projets qui permet de sélectionner les propositions d'action les plus pertinentes et de les subventionner en partie dans le respect des grandes orientations du plan. Ce sont bien les collections qui sont visées par l'appel à projets et non pas les services qui pourraient s'articuler aux réalités patrimoniales. En somme, ces appels reviennent à la réalité organique de la bibliothèque, celle de ses ensembles documentaires qu'il faut ramener à la lumière.

10 Pour prendre la mesure des effets de cette politique incitative, il faut constituer des données chiffrées à partir des résultats des appels à projets. Les sources sont hétéroclites. Les bilans du PAPE sont en ligne pour certaines années, de manière plus ou moins détaillée3. Une enquête a par ailleurs été menée par le Service du livre et de la lecture (SLL) en 2015 pour mesurer les effets du PAPE sur le terrain depuis 20074. Les indicateurs ainsi constitués permettent de donner quelque consistance aux grandes orientations de cette politique, en vérifiant leur application sur le terrain des collections. 
11 Entre 2007 et 2019, 307 projets ont été déposés 5 , soit environ 23 projets par an, les bibliothèques impliquées pouvant en moyenne déposer deux projets, parfois davantage, ce qui révèle désormais un certain esprit de continuité, dans la prise en compte des fonds patrimoniaux. On le voit à la médiathèque publique et universitaire Valence Romans Agglo où, en 2019, le « chantier de conservation préventive des collections patrimoniales » en est à sa " phase 3 : reconditionnement », chacune des phases précédentes ayant été soutenue par le PAPE. Au total, 196 projets ont été retenus en quatorze années. En d'autres termes, $63 \%$ des projets déposés ont convaincu le Service du livre et de la lecture. Ces premiers chiffres indiquent l'existence d'une véritable dynamique locale dans cette régénération des fonds patrimoniaux.

Sur l'identité des bibliothèques concernées, il faut se limiter aux données des années 2007-2015 fournies par l'enquête du ministère de la Culture. Durant cette période, 123 structures différentes ont déposé un projet, et 74 ont vu leur demande exaucée. Élément intéressant à souligner, les bibliothèques municipales classées ne sont à l'origine que de la moitié des projets déposés. Or, les fonds patrimoniaux y jouissent depuis la fin du xixe siècle d'une reconnaissance et de moyens plus importants qu'ailleurs, le classement se fondant précisément sur l'intérêt scientifique des fonds anciens et leur volumétrie. Les bibliothèques non classées se sont donc montrées capables, elles aussi, d'exploiter l'opportunité ouverte par le PAPE et d'ouvrir une nouvelle réflexion sur leurs collections. En revanche, les projets des BMC (bibliothèques municipales classées) ont mieux convaincu le ministère, sans doute parce que les agents y sont plus nombreux et plus capables de formaliser une demande de subvention. Les projets collectifs ne sont pas aussi nombreux que l'appel à projets le souhaite (24 seulement, soit 10,70 \% des demandes), mais ils sont aussi mieux considérés, puisque la majorité d'entre eux ont été retenus. Une cartographie des projets soutenus montre que les bibliothèques des bassins de population plus importants sont avantagées (135 ooo habitants en moyenne), ce qui se traduit concrètement par un plus grand nombre de bibliothèques de capitales régionales parmi les institutions dont les projets ont été retenus. Dijon a obtenu ainsi cinq subventions, Roubaix et Troyes quatre chacune, Albi, Toulouse, Strasbourg et Paris trois chacune. Mais en y regardant de plus près, les Hauts-de-France, l'Occitanie, la Bourgogne et la NouvelleAquitaine sont les quatre régions qui ont obtenu le plus de subventions, au profit de bibliothèques de villes moyennes.

13 Dans ce cadre général, les actions soutenues ont permis aux bibliothèques de revisiter leurs collections. Notre comptage ne prend en compte que les projets concernant les fonds documentaires, et non pas la formation des personnels, également subventionnable, mais qui reste par ailleurs marginale dans les effets du PAPE ${ }^{6}$. Les indicateurs que nous avons constitués montrent la part décroissante de l'aide aux acquisitions, reportée en réalité sur d'autres dispositifs de financement et sur de nouvelles pratiques de mécénat. À l'inverse, ils révèlent la part fortement croissante de l'aide à la valorisation, c'est-à-dire à la prise en compte de nouveaux publics du patrimoine, au-delà du cercle restreint et fidèle des érudits et universitaires ; et, enfin, la part majeure, mais instable, du signalement et de la conservation, qui s'imposent comme le cœur du PAPE. D'autres enquêtes confirment cette tendance (Foucher, $2018:$ 25-26). La typologie des projets déposés, comme celle des projets retenus, montre que le signalement et la conservation sont les mesures phares d'un réveil des fonds patrimoniaux (tableau 1). Il est plus intéressant encore de souligner que plus de la moitié des projets mêlent ces différents plans, indiquant la prise de conscience chez les agents des bibliothèques de la nécessité de reprendre en main le patrimoine de manière globale, en associant connaissance scientifique des fonds, mise en sécurité, diagnostic sanitaire, signalement et identification des publics cibles. Sur le terrain, les coordinateurs du plan, c'est-à-dire, principalement, les conseillers livre et lecture au sein des directions régionales des affaires culturelles (DRAC), constatent eux-mêmes ces progrès. D'après l'enquête menée par le SLL en 2015, les évolutions favorables sont les plus nettes pour le signalement (91,60 \% des réponses), la valorisation (91\%), le 
rayonnement des collections auprès des publics (88 \%), la conservation $(67,40 \%)$ et l'état des locaux (63\%).

Tableau 1. Typologie des projets déposés ou soutenus dans le cadre du PAPE (2007-2019).

\begin{tabular}{|l|l|l|l|}
\hline & $\begin{array}{l}\text { En faveur du } \\
\text { signalement }\end{array}$ & $\begin{array}{l}\text { En faveur de la } \\
\text { conservation }\end{array}$ & $\begin{array}{l}\text { En faveur de la } \\
\text { valorisation }\end{array}$ \\
\hline Projets déposés & $64,30 \%$ & $46,40 \%$ & $46 \%$ \\
\hline $\begin{array}{l}\text { Projets retenus par } \\
\text { le SLL }\end{array}$ & $62,50 \%$ & $44 \%$ & $45,80 \%$ \\
\hline
\end{tabular}

Sources : ministère de la Culture, 2015 (pour les années 2007-2015) ; complété par les résultats des appels à projets 2016, 2017, 2018 et 2019. Un projet peut viser à la fois le signalement et la conservation ou la valorisation.

Concrètement, les projets retenus sont assez homogènes d'une bibliothèque à une autre. Qu'on en juge par les résultats de l'appel de 2018, qui fait état de 22 projets retenus. Le signalement concerne exclusivement les manuscrits, celui des imprimés étant placé sous le regard de la $\mathrm{BnF}$ qui gère des enveloppes budgétaires fléchées. Ainsi, cette année-là, l'agence régionale du livre du Grand Est, Interbibly, a porté un projet collectif des bibliothèques de la région pour la mise à jour de leurs notices dans le Catalogue général des manuscrits des bibliothèques de France, désormais en ligne. La même année, le projet de la bibliothèque de Bayonne, concernant le catalogage rétrospectif des collections iconographiques, a été retenu. La conservation recouvre des projets de restauration (désormais exclus des appels à projets car ils empiétaient sur les prérogatives du Comité technique de restauration), les audits de conservation préventive, les opérations de dépoussiérage, le conditionnement des documents, que ceux-ci soient écrits ou iconographiques. La bibliothèque d'Annonay (Auvergne-Rhône-Alpes) a ainsi bénéficié d'une subvention pour la mise en sécurité sanitaire de ses collections patrimoniales, tandis que celle de Riom a obtenu un soutien financier pour la planification de la mise en sécurité de ses collections patrimoniales dans le cadre de la nouvelle médiathèque intercommunale. La valorisation est jugée pertinente dès lors qu'elle met en valeur un document ou un ensemble documentaire bien identifié, d'un intérêt scientifique supralocal, qu'elle est en rapport avec des commémorations nationales ou un événement de l'histoire de la bibliothèque. Le réseau des médiathèques d'Albi a pu ainsi financer grâce au PAPE le projet de valorisation des manuscrits « D'or et de lumière : quand lettres et lettrines s'animent ».

Ainsi décrit, le PAPE pourrait ressembler à une sorte de laboratoire de bonnes pratiques en matière de signalement et de conservation préventive. Mais cette image n'est que de surface. Il est en réalité l'instrument d'un double projet. Premièrement, il impose une conception du patrimoine comme notion générique et universelle, qui trouve ensuite sa matérialité dans l'éparpillement des collections dans les territoires, celles-ci relevant toutes d'une même matrice nationale. Ensuite, ce patrimoine est une affaire d'État en ce qu'il est la trace d'un être collectif enraciné dans la culture écrite. Conserver n'est pas qu'une affaire technique de contrôle de l'environnement climatique, de dépoussiérage soigneux et d'usage de matériaux neutres. C'est la garantie, promise par l'État, d'une pérennité qu'il doit aux citoyens. Les communes et les intercommunalités ne constituent qu'un intermédiaire dans cette opération symbolique. Signaler n'est pas seulement accommoder des normes descriptives aux documents anciens, même si l'exercice requiert une certaine virtuosité. C'est à la fois permettre une gestion comptable de ce qui fait patrimoine à l'échelle de l'État - ce dont le Catalogue collectif de France (CCFr), en agrégeant les notices ${ }^{7}$, constitue une belle vitrine - et rendre visible cette masse documentaire considérable aux yeux des usagers, voire des bibliothèques elles-mêmes. La politique de l'État en matière de patrimoine des bibliothèques opère ainsi une sorte de révélation au sens photographique du terme. 
Ce retour des collections au centre de l'attention bibliothéconomique est aussi, pour les bibliothèques, un moyen de se singulariser en tant qu'institutions patrimoniales, notamment aux yeux de leurs tutelles qui doivent financer partiellement les projets soutenus par le PAPE. La collection devient ainsi l'étendard de la condition patrimoniale des établissements, non pas seulement par le prestige culturel et scientifique qu'elle confère - la séduction du parchemin, de l'enluminure ou du cuir bien ciré -, mais aussi par sa capacité à réfléchir localement cette vision universaliste du patrimoine. Pour vérifier cette hypothèse, un détour par les documents programmatiques des bibliothèques, dont les règlements des concours du PAPE exigent d'ailleurs l'existence, s'impose.

\section{Les bibliothèques face à leurs collections : quelle argumentation ?}

Depuis bientôt trente ans, le concept de collection en bibliothèque est passé d'une réalité intellectuelle sujette à l'empilement, voire la stratification, à un ensemble arithmétique évaluable et, à ce titre, reconfigurable à volonté (Calenge, 1994, 1999). Les collections patrimoniales n'échappent pas à la règle, avec d'inévitables limites (l'inaliénabilité des collections, en particulier, qui interdit désherbage et éliminations), et font l'objet, comme les autres, de différents documents stratégiques définissant les protocoles de conservation, d'enrichissement et de valorisation.

18 En mars-avril 2020, nous avons lancé un appel aux bibliothèques patrimoniales, par le biais de listes de diffusion d'abord, puis par une série d'appels personnalisés à des échantillons de bibliothèques déterminés selon le type d'établissement et leur implantation géographique. Les 54 bibliothèques municipales classées ont ainsi été toutes sollicitées, et presque autant de bibliothèques non classées, principalement dans les Hauts-de-France, dans le Grand Est, en Normandie et en Occitanie $^{8}$. Au total, 93 établissements ont été contactés, soit un tiers des bibliothèques identifiées par le Service du livre et de la lecture comme conservant des collections patrimoniales 9 . Ces sollicitations, malgré une période peu propice ${ }^{10}$, ont suscité un grand intérêt du côté des directeurs d'établissement et des responsables de fonds patrimoniaux, signe que le sujet n'a rien d'anodin dans les bibliothèques. La sollicitation portait sur l'existence - et, le cas échéant, la communication - d'un Projet culturel, scientifique, éducatif et social (PCSES) pour l'établissement, ou, en sus ou à défaut, de documents stratégiques concernant les collections : chartes documentaires, projets d'établissement, projets de service notamment. Il est intéressant de souligner que sur 57 répondants, deux ne sont pas en mesure de dire s'il existe ou non un PCSES dans leur établissement. Les difficultés de vocabulaire sont aussi nombreuses, mais n'ont guère de sens car un projet d'établissement ou un projet de service peut tenir lieu de PCSES dans la mesure où il comporte nécessairement un volet culturel, éducatif et social (Grandet \& Morel, 2019: 6).

19 Avec un taux de réponse de $61 \%$, notre enquête était peut-être prématurée tant les PCSES, empruntés au monde des musées où ils existent depuis trente ans, sont apparus récemment dans l'univers des bibliothèques. C'est seulement depuis 2012 que le ministère de la Culture a institutionnalisé ces pratiques, voire participé à leur financement. Une enquête sur les PCSES du point de vue de la mise en œuvre d'une démarche " projet » en bibliothèque a souligné également la lenteur de ce processus au sein des bibliothèques (Morel, 2016 : 13). À l'heure actuelle, la rédaction d'un PCSES n'a rien d'obligatoire pour les bibliothèques, le ministère ne pouvant l'exiger de la part de collectivités territoriales.

Vingt et une bibliothèques seulement en disposent dans notre échantillon, et 18 s'apprêtent à en rédiger un ou à le faire valider. Ce sont, aux deux tiers, des BMC. Ce point s'explique facilement par le fait, d'une part que le ministère bénéficie d'une force prescriptive plus grande auprès de ces établissements, et d'autre part que ces établissements sont obligés de se plier à cet exercice dans le cadre de la mise à disposition 
de personnel d'État. Plus généralement, l'écriture d'un PCSES répond souvent à une exigence stratégique de reconnaissance institutionnelle en vue d'obtenir un financement construction d'un nouvel édifice, négociation d'un label de type "Bibliothèque numérique de référence » (BNR) (Duquenne, 2017), par exemple ${ }^{11}$. Pour la bibliothèque-médiathèque intercommunale d'Épinal-Golbey, ainsi, la rédaction (en cours) d'un PCSES a été motivée par l'obtention d'un label BNR et d'un Contrat territoire-lecture (CTL). À Angers, le même exercice, également en cours, est justifié par la rénovation (et probable extension) de la bibliothèque centrale Toussaint, ainsi que plusieurs réaménagements en bibliothèques de quartier. Celui de Chambéry, abouti en 2016, a été rédigé dans le cadre d'une demande de subvention auprès de la DRAC pour la ré-informatisation des bibliothèques. Il est également une préfiguration d'un réseau de bibliothèques à l'échelle de l'agglomération.

Dans cette logique, les collections serviraient alors à démontrer que la bibliothèque peut prétendre à une reconnaissance culturelle - et aux financements assortis - en faisant de sa richesse documentaire, et notamment patrimoniale, un élément de négociation de son rôle politique, social et culturel, discours que les BMC pourraient plus aisément argumenter compte tenu du fait qu'elles bénéficient de cette reconnaissance, au moins juridique et technique. C'est cette hypothèse qu'il faut vérifier.

Avec ces documents, les collections, qui tenaient lieu d'évidence, ont dû être explicitées, argumentées et articulées avec un certain nombre d'impératifs qui indiquent, in fine, que la bibliothèque est d'abord un objet politique et qu'elle est en mesure de se penser comme telle. Le PCSES doit en effet faire de la bibliothèque un moyen pour la collectivité de mettre en œuvre ses priorités. Il est un document engageant et contractuel, qui formalise la relation entre la bibliothèque et sa collectivité ou l'État et transcrit ainsi l'évolution des politiques publiques dans la gestion des bibliothèques. Il est bien certain que dans cette logique, la notion de service est encore prépondérante, puisqu'il s'agit aussi d'identifier des usagers et de leur apporter satisfaction. Mais les collections entrent également dans la gamme des services que la bibliothèque rend à la collectivité et à l'individu, ce qui constitue un glissement notable des attributions des documents patrimoniaux dans l'histoire des bibliothèques. C'est ainsi sous la rubrique des « services » que la bibliothèque municipale à vocation régionale de Nice intègre la question des collections dans son PCSES approuvé en 2018.

Avec l'aide des répondants, nous avons constitué un corpus de 29 PCSES actifs ou presque aboutis. Ces documents, extrêmement détaillés et argumentés, se prêtent bien à une analyse de discours, en s'attachant à repérer le lien sémantique et bibliothéconomique qui lie collection et patrimoine écrit d'une part, la fonction de la collection dans la définition que les bibliothèques proposent d'elles-mêmes dans l'espace politique et social d'autre part. Avec cette limite que le corpus des PCSES est inévitablement mouvant et susceptible d'évolutions très rapides dans les mois et années qui viennent.

24 Il importe d'abord de signaler que plusieurs PCSES n'intègrent pas la composante patrimoniale des collections dans l'argumentaire, y compris ceux de BMC aux fonds très riches. C'est le cas à Périgueux et à Chambéry, où le projet se contente d'évoquer une ressource patrimoniale " qui peut sembler élitiste », précaution rendue nécessaire par le fait qu'il défend une posture de lecture publique étendue à un nouveau réseau. C'est encore le cas à la médiathèque François-Mitterrand de Poitiers, pourtant forte de 500 ooo documents anciens et active dans la valorisation de son patrimoine, mais où la gestion du dossier par un cabinet de consultants (chose habituelle par ailleurs) peut expliquer l'évacuation de la question patrimoniale, ces cabinets étant souvent plus habitués à travailler avec des établissements non patrimoniaux. Établi en 2015, ce projet devait mettre l'accent sur les publics et les services aux usagers. C'est un point important : dans la manière dont la bibliothèque se présente à sa tutelle, les collections font l'objet d'occultation ou de célébration, selon la manière la plus opportune de convaincre les élus de s'appuyer sur la bibliothèque pour construire une politique culturelle. Car ailleurs, ce sont justement les collections patrimoniales qui sont mises en exergue. Ainsi, à Versailles, 
où le patrimoine est quantitativement moins présent (100 ooo documents), le PCSES revendique ${ }^{12}$ :
« Le patrimoine demeure, quant à lui, l'épine dorsale de la Bibliothèque centrale et un enjeu majeur au niveau tant de la conservation que de la médiation qui s'est développée autour au cours des années précédentes. Les actions ciblées ont rencontré leur public, preuve d'un intérêt indiscutable à son égard. La réflexion qui se poursuit sur les collections, notamment celles en magasin, prouve que le patrimoine de demain se construit aujourd'hui et qu'il est important, d'ores et déjà, de se préoccuper de la conservation pérenne de collections intermédiaires. »

Les collections sont alors instrumentalisées en vue de trois propositions : faire du patrimoine le matériau d'une conscience culturelle territoriale ; l'intégrer à la mission éducative de la bibliothèque au prix d'un effort de vulgarisation ; enfin, en faire un objet touristique. Il va de soi que cette typologie n'est pas aussi segmentée que notre propos le suggère : les trois enjeux se conjuguent assez naturellement. Elle tire toutefois sa pertinence de l'argument principal convoqué dans le PCSES sur le chapitre des collections patrimoniales.

La première instrumentalisation est au service de la notion de territoire. Le PCSES de Versailles évoque la « juste échelle » à laquelle le patrimoine peut être rendu vivant, en relation avec l'identité « particulière et prestigieuse » des collections versaillaises, liées à la présence de la famille royale et de la cour sous l'Ancien Régime. À Bayonne, la bibliothèque se veut une vitrine du Pays basque, et le PCSES, validé en 2016, souligne les partenariats régionaux susceptibles de donner corps à un consensus territorial : dans le domaine éducatif, avec le rectorat ; dans le domaine touristique, avec les offices du tourisme ; etc. La bibliothèque municipale (classée) de Nancy, qui partage avec les bibliothèques de Metz, Épinal et Thionville un projet culturel et scientifique commun en vue d'une labellisation BNR, défend avec elles la mise en exergue des « cultures lorraines » comme forme de connivence entre les habitants de ce vaste axe régional qui s'étend de la frontière luxembourgeoise aux portes du massif vosgien. Chaque bibliothèque doit déterminer au sein de ses collections les corpus susceptibles de conforter ces « cultures lorraines ». À Rennes, à la bibliothèque des Champs Libres :

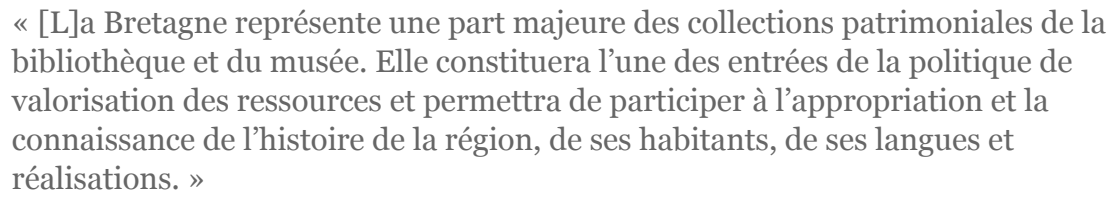

Dans un projet quinquennal (2018-2022) par ailleurs entièrement fondé sur l’idée de capitaliser et partager des connaissances, la bibliothèque assume ainsi une fonction redistributive, y compris à partir des collections patrimoniales. Voire, une fonction participative, selon une posture bibliothéconomique qui connaît désormais un grand succès (Bats, 2015). À Venelles, en Région Sud, la médiathèque est dépourvue de collections patrimoniales, mais sa direction souhaite en constituer à partir d'un " wiki » à l'usage des nouveaux arrivants, et fondé sur la collecte de témoignages, de vidéos, d'archives privées, de photographies et d'enregistrements sonores, afin de créer une culture commune. Ce parti pris régionaliste ou urbain répond d'ailleurs le plus souvent à un besoin local. À Roubaix, les collections patrimoniales sont mises au service d'une " exigence d'image », tant la ville et son histoire souffrent d'un déficit de considération. Les collections peuvent ainsi donner aux habitants des outils pour améliorer la réputation d'une ville de tradition ouvrière supposée en crise : " à Roubaix plus qu'ailleurs, on a besoin du patrimoine », conclut le PCSES formalisé en 2018.

28 Cette posture régionalisante peut paraître élémentaire, voire convenue, mais le fait que d'autres établissements lui tournent délibérément le dos montre que ce n'est pas le cas. À Pau, l'attractivité des collections (120 000 documents) est revendiquée à l'échelle nationale plutôt que régionale : 
« Au niveau national, il s’agit aussi de révéler le patrimoine écrit de l’agglomération en donnant largement accès aux collections de la bibliothèque patrimoniale, de rendre visibles ses trésors, mais aussi de mieux les signaler pour à la fois préserver et faire connaître un patrimoine méconnu. »

C'est plus net encore à Tours où le PCSES se fonde sur le statut de bibliothèque municipale classée et donc sur l'importance scientifique reconnue des collections à l'échelle nationale. Cet argument est particulièrement utilisé à propos des collections jeunesse, la ville pouvant se targuer d'avoir hébergé l'une des premières bibliothèques de l'Heure Joyeuse, à partir de 1937. Le PCSES formule ainsi deux ambitions :
« Positionner la bibliothèque municipale de Tours comme l'un des lieux ressources dans le domaine de la littérature pour la jeunesse à l'échelle de la ville, de la région, du territoire national, en donnant une meilleure visibilité à nos fonds riches et uniques, et en permettant ainsi un large rayonnement. Valoriser un héritage, fruit de l'histoire de la bibliothèque de Tours en matière de politique de lecture à destination de la jeunesse. »

Un deuxième groupe de bibliothèques adosse les collections à la mission éducative de l'établissement. À Bordeaux, le patrimoine écrit et graphique est présenté comme un vecteur de partage et d'ouverture, les valeurs fondamentales sur lesquelles la bibliothèque se positionne : d'abord parce qu'il intéresse tout le monde ; ensuite parce que par le biais du numérique, il transcende les divisions traditionnelles entre la lecture publique et l'érudition. Il doit donc être « mis à la portée de tous » et peut devenir un facteur efficace de vivre ensemble. Le PCSES (2016-2020) expose :

« Le patrimoine écrit et graphique, de par la profondeur historique qu'il apporte et que les bibliothécaires s'efforcent de transmettre à un large public, est de nature à rassembler et à contribuer à l'éducation à la citoyenneté : soit parce qu'il conforte un sentiment d'appartenance tout en l'expliquant et en le remettant dans son contexte, soit parce qu'il permet de mieux comprendre les événements du présent. »

Ce lien nouveau entre patrimoine et lecture publique cherche visiblement à résoudre, dans la culture professionnelle bibliothécaire comme dans la culture politique des élus, l'écartèlement originel qui a tant desservi les bibliothèques. On le voit à Rochefort, en Nouvelle-Aquitaine, où le PCSES, qui court de 2019 à 2023, affiche une volonté de désacraliser le patrimoine en affirmant :
« Le fonds patrimonial de la médiathèque de Rochefort fait partie des atouts majeurs du réseau 'M. Trésor patrimonial comptant des pièces uniques et de nombreux liens avec les collections des musées de Rochefort, il doit pouvoir être relié par l'action culturelle aux ressources et actions proposées en lecture publique. »

Le PCSES de la bibliothèque Inguimbertine de Carpentras, qui, par sa précocité, a longtemps servi de modèle pour les bibliothèques de la Région Sud, voire de France (Delmas, 2008, 2011), cherche aussi à réduire l'écart entre lecture publique et patrimoine dans la description qu'il donne du futur équipement (inauguré depuis, en 2017), en évoquant un «partage culturel » et en signifiant que « cette valorisation patrimoniale ne suffit pas à elle seule à répondre à toutes les préoccupations de la lecture publique, mais [qu']en proposant des supports variés de connaissance, elle peut contribuer à la développer ». Les collections anciennes ou contemporaines sont censées participer de la même manière au développement de la lecture si elles sont accompagnées de mesures de médiation. À une échelle plus modeste, le projet de la bibliothèque de Verdun (2019) entend réconcilier aussi lecture publique et patrimoine en faisant circuler les collections de la bibliothèque d'étude (50 ooo documents), située en ville haute, vers la médiathèque sise, elle, en ville basse.

Ainsi récupérées, les collections se prêtent à un troisième niveau de valorisation politique, qui emprunte aux deux précédents : leur inclusion dans une stratégie touristique. À Saint-Dié-des-Vosges, alors que les collections sont restées en déshérence 
pendant des décennies, un nouveau projet de bâtiment a donné lieu à un PCSES (2018), qui s'appuie sur le fait que le nouvel édifice sera partagé avec l'office du tourisme. Les collections patrimoniales (90 ooo documents) seront valorisées dès l'espace dédié à l'OT à travers un dispositif muséographique interactif et numérique. "Véritable vitrine pour le territoire, les collections patrimoniales bénéficieront alors d'une visibilité accrue pour les touristes. » Cet impératif touristique s'appuie sur la réputation du Festival international de géographie qui se déroule annuellement dans la petite cité vosgienne et qui paraît une opportunité intéressante pour la direction de la bibliothèque comme pour les élus. À une échelle territoriale plus vaste, les médiathèques d'Albi revendiquent aussi de "fédérer autour d'une destination culturelle et touristique de référence, d'une offre patrimoniale d'excellence ». Cette proposition implique notamment un grand chantier d'identification de la composante albigeoise des collections, dans une logique de discrimination régionale. À Brest, l'ambitieux projet de rénovation des ateliers maritimes des Capucins en médiathèque, entamé en 2010, reposait sur un projet scientifique et culturel qui a mis le patrimoine au cour d'une problématique urbaine rapprochant intentionnellement la cinémathèque, les archives, la bibliothèque et différentes structures associatives vouées à l'histoire et à la création artistique. Les collections patrimoniales de ces différents pôles, d'après ce projet, devaient composer un " centre d'interprétation » dédié au patrimoine brestois et aux identités littorales. La réserve précieuse devait être visitable, pour répondre à une demande touristique grandissante à l'égard des bibliothèques. Un espace muséographique voisin de la salle de consultation des collections était prévu. Un tel dispositif devait transformer les collections patrimoniales en objet touristique. La bibliothèque, inaugurée en 2017, est restée finalement en deçà du projet défendu dans le PCSES, et le centre d'interprétation n'a pas vu le jour. Mais ce projet brestois attire notre attention sur la séduction qu'opère sur les collectivités territoriales la muséification des bibliothèques à partir de certaines strates de collections. La réserve précieuse est visitable à Épinal ; la bibliothèque-médiathèque Grand Troyes a développé un espace d'exposition permanente qui permet au visiteur une véritable déambulation chronologique dans les collections ; la Bibliothèque humaniste de Sélestat est désignée par la ville comme un lieu de tourisme, susceptible de renforcer l'attractivité de la petite cité alsacienne (Naas, 2016). Il est intéressant que de tels discours soient produits au sein même des bibliothèques, car au niveau de l'État, les instrumentalisations politiciennes du patrimoine, c'est-à-dire « la tentation pour certains élus d'utiliser l'image patrimoniale des collections pour la communication de la ville, du département et de la région » (Loiret, $2014: 18-19$ ), sont perçues comme une possible distorsion de la composante patrimoniale de la bibliothèque, qui ne saurait être réduite à une série de stéréotypes commodes en rapport avec l'identité locale. Or, ce sont ici les bibliothèques elles-mêmes qui proposent ces instrumentalisations, en se promettant sans doute de les maîtriser.

D'un point de vue opérationnel, une lecture attentive des PCSES et documents proches permet d'élaborer un palmarès des procédures bibliothéconomiques qui permettront d'atteindre ces objectifs. La plus importante est indiscutablement la numérisation, évoquée dans la totalité des projets, qu'elle soit déjà massive dans la bibliothèque, ou seulement balbutiante ${ }^{13}$. La bibliothèque municipale de Versailles espérait une labellisation BNR (obtenue en 2018) ; la médiathèque intercommunale de Pau, pour son projet 2019-2023, entend s'appuyer sur un partenariat avec la BnF pour mettre en place un portail dédié à ses collections numérisées « qui assurera la visibilité des collections numériques en région mais aussi à l'échelle internationale ». À Saint-Étienne, la numérisation doit être concertée avec les autres équipements culturels de la ville (notamment pour le vaste ensemble des collections de cartes postales), pour plus de cohérence et de visibilité. Cette frénésie de numérisation, pour légitime qu'elle soit à bien des égards, pose justement des questions fondamentales en termes de collections. L'opération technique qu'elle représente ne consiste pas seulement à faciliter l'accès aux documents et à constituer un " témoin numérique » (selon le PCSES de la bibliothèque de Versailles) ou un miroir des collections physiques, mais elle crée de nouveaux documents 
et de nouvelles collections, qui exigent d'autres modalités de conservation, de signalement et de médiation (Chevry, 2011).

35 La conservation préventive constitue le second protocole consensuel autour des collections. Outre qu'elle paraît rassurante aux yeux des élus et représente la fonction la plus traditionnelle du bibliothécaire, elle relève, on l'a vu, d'une véritable urgence. Les bibliothèques de Bordeaux, de Tours, de Saint-Dié, d'Albi, d'Épinal, de Lyon, d'Orléans ou de Clermont-Ferrand y font explicitement référence. Enfin, de manière beaucoup plus diffuse, la valorisation est présentée comme un gage de visibilité non seulement des collections, mais aussi de la fonction de la bibliothèque dans la cité comme garante d'un passé commun. À Nîmes, le développement de l'offre documentaire régionale doit aboutir à « la constitution d'une vraie collection locale de référence et, à plus long terme, la création d'un portail de l'histoire locale ", faisant de la bibliothèque un lieu d'expertise historique. La bibliothèque de Versailles accueille pour sa part des chercheurs en résidence, qui accèdent aux collections à volonté du moment qu'ils traitent un sujet intéressant le territoire (sous le regard des agents du fonds patrimonial), puis restituent leur recherche dans différents formats auprès de différents cercles de public. À Bordeaux, la bibliothèque entend organiser tous les deux ans un événement phare autour des collections patrimoniales, mais d'envergure nationale. À Albi, la médiathèque souhaite mettre en place un service de questions-réponses sur la culture régionale à partir des collections patrimoniales, avec l'aide des autres équipements culturels albigeois.

Aucun de ces PCSES ne prend position sur une politique d'enrichissement - c'est, il est vrai, plutôt le rôle des chartes documentaires. Une étude précise des acquisitions patrimoniales des bibliothèques depuis trente ans montrerait justement que les bibliothèques municipales ont élaboré, avec la complicité des élus et des services de l'État, des priorités patrimoniales en cohérence avec le triple positionnement que nous avons décrit. Les aides financières des régions et de l'État, complétant l'investissement des villes dans ces opérations, visent précisément des biens patrimoniaux qui soutiennent l'identité régionale, à travers le panthéon littéraire local (les archives et manuscrits d'écrivains), la photographie et la carte postale, et, enfin, les manuscrits médiévaux et modernes de production ou d'usage local (Deville, 2000 ; Saint-Marc, 2019), au risque, parfois, d'inventer de toutes pièces une identité urbaine ou régionale. À travers ces enrichissements, mais aussi au fil des gestes bibliothéconomiques et des retours que la bibliothèque opère sur elle-même dans ses documents-cadres, les collections sont devenues ce qu'elles n'avaient peut-être même pas été à l'origine de ces équipements culturels : des objets de désir, de curiosité, de prospection scientifique nourrissant aussi la légitimité de ces institutions.

\section{Conclusion}

C'est donc peu de dire qu'à l'échelle municipale ou intercommunale, les bibliothèques ont construit un discours qui prend les collections pour prétexte et pour argument d'une nouvelle définition de leur rôle, assumé ensuite à travers des priorités qui convoquent des gestes bibliothéconomiques traditionnels ou innovants. Au-delà des mesures de conservation et de signalement, c'est un nouvel univers des collections qui se dessine, et qui, s’il emprunte logiquement aux priorités de l'État en la matière, ne s'y superpose pas totalement. La question du signalement est ainsi évacuée, ou seulement implicite, dans nombre de PCSES alors que le ministère de la Culture en a fait une priorité depuis l'origine du PAPE. Ce n'est pas prendre les bibliothèques en flagrant délit de contradiction. C'est simplement observer que le même équipement, selon qu'il espère le soutien financier de l'État et, à travers lui, une reconnaissance de sa réputation patrimoniale, ou selon qu'il tente de reconstruire ses liens politiques avec la collectivité qui le finance, n'utilise pas les collections de la même manière. Vis-à-vis du premier, il s'agit de dénombrer, décrire et protéger un patrimoine au sens juridique du terme, patrimoine national qui plus est. Vis- 
à-vis de la seconde, il s'agit de réinventer le patrimoine comme réalité documentaire locale et de le recharger de sens nouveaux, diffus, suffisamment efficaces pour susciter l'adhésion des élus. Le retour aux collections précieuses n'est pas seulement motivé par la recherche d'identités locales ou par la séduction de l'ancien et le mystère de cette " part cachée » de l'institution, il s'impose, comme le suggérait déjà Jean-Paul Oddos il y a plus de vingt ans, pour renouveler la fonction même de la bibliothèque (1997 : 13).

C'est que, comme " rouage » d'une collectivité, les bibliothèques ne sont aussi que l'« outil » (Lahary, 2015) de politiques publiques pour lesquelles elles peuvent être force de proposition - les PCSES le prouvent - mais, avant tout, un instrument aux mains d'un exécutif. Sommées de prouver leur utilité régulièrement remise en cause, les bibliothèques ont trouvé dans leurs réserves patrimoniales un moyen à la fois individuel et local, mais aussi collectif et national, de prouver qu'elles sont irremplaçables. Les collections sont bien le meilleur atout des bibliothèques pour assurer leur avenir.

\section{Bibliographie}

Bats (Raphaëlle). 2015. Construire des pratiques participatives dans les bibliothèques. Villeurbanne : Presses de l'Enssib.

DOI : 10.400o/books.pressesenssib.4137

Calenge (Bertrand). 1994. Les Politiques d'acquisition. Constituer une collection dans une bibliothèque. Paris : Éditions du Cercle de la librairie.

Calenge (Bertrand). 1999. Conduire une politique documentaire. Paris : Éditions du Cercle de la librairie.

Chevry (Emmanuelle). 2011. Stratégies numériques : Numérisation et exploitation du patrimoine écrit et iconographique. Paris : Hermes Science - Lavoisier (Traitement de l'information).

Cohen (Gérard) \& Yvon (Michel). 2004. " Le Plan d'action pour le patrimoine écrit ». Bulletin des bibliothèques de France [en ligne], 5, p. 48-50 : https://bbf.enssib.fr/consulter/bbf-2004-05-0048008 [consulté le 5 novembre 2020].

Delmas (Jean-François). 2008. « Carpentras : le projet scientifique et culturel de l'Inguimbertine ». Patrimoines. Revue de l'Institut national du patrimoine, 4, p. 56-63.

Delmas (Jean-François). 2011. « Le projet scientifique et culturel de l'Inguimbertine. Un exemple d'approche muséale au service des bibliothèques ». Bulletin des bibliothèques de France [en ligne], 4, p. 26-31 : https://bbf.enssib.fr/consulter/bbf-2011-04-0026-005 [consulté le 5 novembre 2020].

Desgraves (Louis) (dir.). 1982. Le Patrimoine des bibliothèques. Rapport à Monsieur le Directeur du livre et de la lecture par une commission de douze membres. Paris : ministère de la Culture.

Deville (Jacques). 2000. "Les Bibliothèques dans le marché du patrimoine écrit et graphique ». Bulletin des bibliothèques de France [en ligne], 2, p. 52-62 : https://bbf.enssib.fr/consulter/bbf2000-02-0052-007 [consulté le 5 novembre 2020].

Duquenne (Isabelle). 2017. "Vers un aménagement numérique du territoire ». Bulletin des bibliothèques de France [en ligne], 12, p. 44-51 : https://bbf.enssib.fr/consulter/bbf-2017-12-0044005 [consulté le 5 novembre 2020].

Evans (Christophe), Maresca (Bruno) \& Gaudet (Françoise). 2013. Les Bibliothèques municipales en France après le tournant Internet. Attractivité, fréquentation et devenir. Paris : Éditions de la Bibliothèque publique d'information.

Foucher (Cécile-Tiphaine). 2018. Pour que vive le patrimoine écrit. Démocratiser son accès. Mémoire pour le diplôme de conservateur de bibliothèque, Villeurbanne, Enssib.

Gautier-Gentès (Jean-Luc). 1998. " Le contrôle de l'État sur le patrimoine des bibliothèques des collectivités et des établissements publics », p. 55-70 in Rapport annuel 1997. Paris : Inspection générale des bibliothèques.

Grandet (Odile) \& Morel (Anne). 2019. Concevoir et faire vivre un projet d'établissement en bibliothèque. Villeurbanne : Presses de l’Enssib.

Henryot (Fabienne) (dir.). 2019. La Fabrique du patrimoine écrit : Objets, acteurs, usages sociaux. Villeurbanne : Presses de l'Enssib.

Kupiec (Anne). 1997. Le Livre-sauveur. La question du livre sous la Révolution française, 17891799. Paris : Kimé. 
Lachal (Jérémy). 2019. "Les bibliothèques au cœur de la transformation sociale ». Documentation et bibliothèques, 65(1), p. 12-16.

DOI : $10.7202 / 1061815$ ar

Lahary (Dominique). 2015. "Les bibliothèques au risque des politiques publiques ». Bulletin des bibliothèques de France [en ligne], $\mathrm{n}^{\circ}$ 5, p. 54-70 : https://bbf.enssib.fr/consulter/bbf-2015-050054-006 [consulté le 6 novembre 2020].

Lassalle (Marine de). 1997. « Les Paradoxes du succès d'une politique de lecture publique ». Bulletin des bibliothèques de France [en ligne], $\mathrm{n}^{\circ}$ 4, p. 10-17 : https://bbf.enssib.fr/consulter/bbf-1997-040010-002 [consulté le 5 novembre 2020].

Loiret (Manuella). 2014. Ambitions et mise en œuvre d'une politique en faveur du patrimoine écrit en région : Essai de bilan du PAPE (2004-2012). Mémoire pour le diplôme de conservateur de bibliothèque, Villeurbanne, Enssib.

Morel (Anne). 2016. PSC/PCSES : Pour une diffusion de la démarche projet en bibliothèque. Mémoire pour le diplôme de conservateur de bibliothèque, Villeurbanne, Enssib.

Naas (Laurent). 2016. "Une bibliothèque en cours de métamorphose : vers la Nouvelle Bibliothèque humaniste », p. 183-202 in Gutenberg Jahrbuch 2016 [91e année] / sous la direction de Stephan Füssel. Wiesbaden : Harrassowitz Verlag.

Oddos (Jean-Paul) (dir.). 1997. Le Patrimoine : Histoire, pratiques et perspectives. Paris : Éditions du Cercle de la librairie.

Plazannet (Fabien). 2008. "Le Plan d'action pour le patrimoine écrit : coordonner, accompagner, évaluer ». Bulletin des bibliothèques de France [en ligne], 6, p. 14-19 : https://bbf.enssib.fr/consulter/bbf-2008-06-0014-002 [consulté le 2 novembre 2020].

Poulain (Martine) (dir.). 1992. Histoire des bibliothèques françaises. IV : Les Bibliothèques au xxe siècle : 1914-1990. Paris : Promodis / Éditions du Cercle de la librairie.

Saint-Marc (Manon). 2019. Quelles politiques de soutien aux acquisitions patrimoniales des bibliothèques territoriales ? Mémoire pour le diplôme de conservateur de bibliothèque, Villeurbanne, Enssib.

Servet (Mathilde). 2010. " Les bibliothèques troisième lieu : une nouvelle génération d'établissements culturels ». Bulletin des bibliothèques de France [en ligne], 4, p. 57-63 : https://bbf.enssib.fr/consulter/bbf-2010-04-0057-001 [consulté le 2 novembre 2020].

Tesnière (Valérie). 2006. " Patrimoine et bibliothèques en France depuis 1945 ». Bulletin des bibliothèques de France [en ligne], $\mathrm{n}^{\circ}$ 5, p. 72-80 : https://bbf.enssib.fr/consulter/bbf-2006-050072-002 [consulté le 5 novembre 2020].

Varry (Dominique). 1989. " "Il faut que les lumières arrivent par torrents.” La Révolution française et la création des bibliothèques publiques : projets et réalités ». Bulletin des bibliothèques de France [en ligne], 2-3, p. 160-165 : https://bbf.enssib.fr/consulter/bbf-1989-02-0160-010 [consulté le 5 novembre 2020].

Varry (Dominique). 1990. " Plaidoyer pour l'inventaire des fonds patrimoniaux ». Bulletin des bibliothèques de France, 2, p. 99-103 : https://bbf.enssib.fr/consulter/bbf-1990-02-0099-003 [consulté le 5 novembre 2020].

Varry (Dominique) (dir.). 1991. Histoire des bibliothèques françaises. III : Les Bibliothèques de la Révolution et du XIXe siècle : 1789-1914. Paris : Promodis / Éditions du Cercle de la librairie.

\section{Annexe}

Liste des PCSES (Projet culturel, scientifique, éducatif et social) aimablement transmis par les bibliothèques municipales en mars-avril 2020 :

Albi, réseau des médiathèques de l'Albigeois : Projet scientifique et culturel (2018).

Bayonne, médiathèque de Bayonne : Projet scientifique, culturel, éducatif et social 2016 (2016).

Bordeaux, Ville de Bordeaux, Direction des bibliothèques, de la lecture publique et des médias culturels : Projet culturel et scientifique 2016-202O (2016).

Brest, Ville de Brest, médiathèque des Capucins : Projet scientifique et culturel (2011).

Cambrai : Le Laboratoire culturel de Cambrai. Projet (sans date).

Carpentras : L'Inguimbertine à l'hôtel-Dieu. Rapport sur le Projet scientifique et culturel du Service bibliothèque-musée de Carpentras (2009).

Châlons-en-Champagne : Projet de service du réseau des bibliothèques de Châlons-en-Champagne 2018-2O2O (2018). 
Épinal, bibliothèque-médiathèque intercommunale Épinal-Golbey : Plan pluriannuel de la BMI en matière de gestion et de valorisation du patrimoine écrit 2019-2022 (2019).

Nancy, le Sillon lorrain, pôle métropolitain : Bibliothèque numérique de référence du Sillon lorrain 2013-2015 (2013).

Nice, Ville de Nice, bibliothèque municipale à vocation régionale de Nice : Projet culturel et scientifique 2018 (2018).

Nîmes : Projet culturel et scientifique de la bibliothèque de Nîmes 2018-2022 (2018).

Orléans : Axes du projet d'établissement 2018-2022 (2018).

Pau, réseau des médiathèques de la communauté d’agglomération Pau-Béarn-Pyrénées : Stratégie de développement de la lecture publique 2019-2023 (2019).

Périgueux, médiathèque Pierre-Fanlac : Projet scientifique et culturel 2016-2019 (2016).

Poitiers, médiathèque François-Mitterrand : Étude portant sur la place et le rôle de la médiathèque et son réseau sur le territoire de la ville de Poitiers et du Grand Poitiers. Rapport pour le comité stratégique du 15 décembre 2015.

Rennes, les Champs Libres : Projet culturel et scientifique 2018-2022 (2018).

Rochefort-Océan, communauté d'agglomération Rochefort-Océan, réseau des médiathèques : Projet culturel, scientifique, éducatif et social 2019-2023 (2019).

Roubaix : La Grand-Plage, BMC/BNR (2019).

Saint-Étienne : Projet de service de la médiathèque municipale de Saint-Étienne 2014-2020 (2014).

Saumur, Saumur Val de Loire agglomération, L’Imagin'R, réseau des bibliothèques : Schéma directeur de la lecture publique 2018-2023 (2018).

Tours, Ville de Tours : Projet scientifique et culturel des bibliothèques de Tours 2017-2021 (2017).

Venelles, bibliothèque municipale de Venelles : Projet scientifique, culturel, éducatif et social de la médiathèque de Venelles (2016).

Verdun : Projet de médiathèque du Grand Verdun «L'Æncre ». Note d'intention (2019).

Versailles : Bibliothèques municipales classées de Versailles 2018-2023 : rénover et innover (2018).

\section{Notes}

1 En ligne : http://bbf.enssib.fr/ (consulté le 2 novembre 2020). Les statistiques qui suivent ont été réalisées par nos soins à partir de 2009.

2 Code général de la propriété des personnes publiques, art. L. 2112-1, 10 ${ }^{\circ}$, en ligne : https://www.legifrance.gouv.fr/codes/section_lc/LEGITEXTooooo6070299/LEGISCTA0oooo6164223/200805-05/\#LEGIARTIOOOO06361198 (consulté le 2 novembre 2020).

3 Pour 2018 : http://www.ar2l-hdf.fr/upload/medias/rsultats-aap-2018-sur-site-pdb.pdf (consulté le 2 novembre 2020) ; pour 2019 : https://www.culture.gouv.fr/Sites-thematiques/Livre-etLecture/Patrimoine-des-bibliotheques/Politiques-patrimoniales-de-l-Etat/Plan-d-action-pour-lepatrimoine-ecrit2 (consulté le 2 novembre 2020).

4 « Les dix ans du Plan d'action pour le patrimoine écrit : essai de bilan (très) partiel », ministère de la Culture et de la Communication, Service du livre et de la lecture, 2015, en ligne : http://www.culture.gouv.fr/content/download/134950/1452584/version/2/file/Pr\%C3\%A9sentation\%20PAPE\%20MAD.pdf (consulté le 2 novembre 2020).

5 Nous cumulons ici les chiffres fournis par le rapport de 2015 et ceux qui figurent dans les synthèses de 2018 et 2019, la première comportant un volet rétrospectif pour 2016 et 2017.

6 Pour être complet, il faut expliquer l'absence des projets de numérisation dans le concours : ceuxci peuvent bénéficier d'autres enveloppes et d'autres cadrages, en particulier le label « Bibliothèques numériques de référence » qui, s'il ne se fonde pas sur les fonds patrimoniaux, consacre principalement ceux-ci dans les faits.

7 En avril $2020: 7$ millions de notices.

8 Ces choix se fondent surtout sur la commodité (variable) des annuaires des agences régionales du livre pour identifier les directrices et directeurs d'établissement ; nous avons exploité ceux qui fournissent systématiquement les noms et adresses électroniques de ces personnes.

9 D’après les listes aimablement fournies par Mme Catherine Granger, directrice du Bureau du patrimoine au Service du livre et de la lecture, que je remercie. 
10 Le confinement pour endiguer l'épidémie de Covid-19 a été décrété le 17 mars 2020, et il était encore actif quand nous avons achevé cette enquête. Les personnels des bibliothèques se trouvaient donc en télétravail, avec des priorités changeantes et un accès inégal à leurs dossiers de travail. Je veux remercier ici les 57 établissements qui se sont donné la peine d'une réponse détaillée à ma demande.

11 Sur ce dispositif, voir en ligne : https://www.culture.gouv.fr/Sites-thematiques/Livre-etLecture/Bibliotheques/Numerique-et-bibliotheques/Les-Bibliotheques-numeriques-de-reference (consulté le 5 novembre 2020). La bibliothèque candidate doit présenter un PCSES pour que son dossier soit examiné.

12 L'ensemble des citations qui suivent sont empruntées aux PCSES qui nous ont été communiqués.

13 En juin 2020, 13 BMC sur 54 ne disposent pas d'une bibliothèque numérique patrimoniale en propre, ce qui n'exclut pas qu'elles participent à des programmes collectifs de numérisation, notamment dans le domaine de la presse ancienne. Parmi ces 13 bibliothèques figurent 5 bibliothèques dotées d'un PCSES et/ou d'un projet de BNR. Le délai de mise en œuvre d'un plan est donc assez long.

\section{Pour citer cet article}

Référence papier

Fabienne Henryot, « Les collections, nouvel atout des bibliothèques », Culture \& Musées, 37 | 2021, 135-162.

Référence électronique

Fabienne Henryot, « Les collections, nouvel atout des bibliothèques », Culture \& Musées [En

ligne], 37 | 2021, mis en ligne le 01 juin 2021, consulté le 30 juin 2021. URL :

http://journals.openedition.org/culturemusees/6343; DOI :

https://doi.org/10.4000/culturemusees.6343

\section{Auteur}

\section{Fabienne Henryot}

Enssib, Centre Gabriel Naudé

Fabienne Henryot est maîtresse de conférences HDR à l'Enssib, Centre Gabriel Naudé. Elle est spécialiste de l'histoire du livre et des bibliothèques des époques moderne et contemporaine, et des processus de patrimonialisation de l'écrit. Elle a publié notamment: L'Historien face au manuscrit. Du parchemin à la bibliothèque numérique (Presses universitaires de Louvain, 2012) ; Livres et lecteurs dans les couvents mendiants, Lorraine, $x^{\prime} e_{-x V I I}{ }^{e}$ siècle (Droz, 2013) ; Bibliothèques, religions, laïcité (Maisonneuve \& Larose / Hémisphères, 2018); et La Fabrique du patrimoine écrit. Objets, acteurs, usages sociaux (Presses de l'Enssib, 2020).

Courriel : fabienne.henryot[at]enssib.fr

\section{Droits d'auteur}

Culture \& Musées 